function. Erectile dysfunction in SHRs may be due to oestradioltestosterone imbalance.

\section{GW23-e1799 EFFECTS OF VALSARTAN AND BENAZAPRIL ON SERUM SEX HORMONES, GONADO-SOMATIC INDEX AND ERECTION FUNCTION IN SPONTANEOUSLY HYPERTENSIVE RATS}

doi:10.1136/heartjnl-2012-302920b.30

${ }^{1}$ Liu Huangjun, ${ }^{1}$ Liu Xueyin, ${ }^{2}$ Liu Zhenlan, ${ }^{3}$ Fan Wen, ${ }^{3}$ Zhang Jiajun, ${ }^{4}$ Li Haiping,

${ }^{4}$ Zou Qifu, 'Lei Ru, ${ }^{1}$ Yang Xuexin, ${ }^{5}$ Chen Ming, ${ }^{1}$ Jin Lijun. ${ }^{1}$ Department of Cardiology, The First Affiliated Hospital of Yangtze University; ${ }^{2}$ Department of Gynaecology, The First Affiliated Hospital of Yangtze University; ${ }^{3}$ Clinical Laboratory, The First Affiliated Hospital of Yangtze University; ${ }^{4}$ Department of Nuclear Medicine, The First Affiliated Hospital of Yangtze University; ${ }^{5}$ Medical central laboratory, The First Affiliated Hospital of Yangtze University

Objectives To evaluate the effects of ACE inhibitor and angiotensin receptor blocker on serum sex hormones, gonado-somatic index (GSI) and erection function in spontaneously hypertensive rats (SHRs).

Methods 24 SHRs were distributed initially in three groups: Valsartan group $(n=8)$ received valsartan $(30 \mathrm{mg} / \mathrm{kg} /$ day $)$ and Benazapril group $(n=8)$ received benazapril $(10 \mathrm{mg} / \mathrm{kg} /$ day $)$ dissolved in $0.5 \mathrm{ml}$ distilled water, the Control group $(\mathrm{n}=8)$ received only distilled water $(0.5 \mathrm{ml} /$ day). Then 8 Wistar-Kyoto rats (WKYs) (WKY group) were treated with the same as Control group. Body weight, systolic blood pressure (SBP) and sex hormones were measured at baseline and after treatment in all rats. The intracavernous pressure (ICP) and mean arterial pressure (MAP) were measured at the end of treatment course. Then the rats were sacrificed by carotid artery bloodletting, serum was stored to measure sex hormones by electro chemiluminescence assay. The testicles were removed for measuring testicular weight quickly.

Results Both valsartan and benazapril reduced the SBP after treatment with 2 months, but serum testosterone and oestradiol weren't reduced. As compared with Valsartan and Benazapril group, the testosterone of Control group was reduced and the oestradiol was increased $(p<0.05)$. In addition, pregnendione of the Control group was lower than WKY group $(p<0.05)$ but no significant difference was found during other groups. The ICP/MAP of Control group was lower than the other three groups at 0,2 , $5 \mathrm{~V}$ of cavernous nerve electrostimulation $(\mathrm{p}<0.001)$, which in the WKY group was higher than Valsartan and Benazapril group $(p<0.001)$, the Benazapril group $(p<0.05)$ and there was no significant difference in the three groups $(p>0.05)$ at $0,2,5 \mathrm{~V}$ of electrostimulation, respectively. No significant difference of testicle weight and GSI were found in all the groups $(p>0.05)$.

Conclusions The serum testosterone was reduced and the oestradiol was increased in the control SHRs, which harm to the erectile 